\title{
Der Internationale Strafgerichtshof in Den Haag - Ein Plädoyer für kriminologische Einmischung
}

\section{Frank Neubacher}

\section{Ein langer Weg}

$\mathrm{D}$ ie Kriminologie beschäftigt sich als Erfahrungswissenschaft vom abweichenden Verhalten und seiner Kontrolle mit Erscheinungsformen, Ursachen und Reaktionen auf Kriminalität. ${ }^{1}$ Deshalb wäre zu erwarten, dass sie sich auf breiter Front und entschieden auch den jüngsten Entwicklungen zuwendet, die zur Errichtung des ständigen Internationalen Strafgerichtshofes in Den Haag geführt haben. Denn wer würde bestreiten, dass es sich bei Genozid, Verbrechen gegen die Menschlichkeit $^{2}$ und Kriegsverbrechen, die auch als Kernverbrechen (core crimes) oder als Menschheitsverbrechen bezeichnet werden, um schwerste Kriminalität handelt? ${ }^{3}$ - So schwerwiegend immerhin, dass über Jahrzehnte hinweg weder die Vereinten Nationen noch die internationale Gemeinschaft irgendeinen Zweifel an der Strafwürdigkeit dieser Taten gelassen haben. Wer jedoch, ausgestattet mit dieser Erwartungshaltung, das kriminologische Schrifttum der letzten Jahre mustert, wird enttäuscht. Vom viel zitierten Referenzwerk Herbert Jägers abgesehen ${ }^{4}$, welches entstand, als es noch den Eisernen Vorhang gab, nur beredtes Schweigen. Erst in jüngster Zeit scheint eine neue Generation, gleichermaßen inspiriert durch Jäger wie durch den Strafgerichtshof, die Thematik für die Kriminologie zu entdecken. ${ }^{5}$ Das Völkerstrafrecht ist da schon viel weiter. Es hat die Entstehung des Römischen Statuts zur Errichtung des Internationalen Strafgerichtshofs vom 17.7.1998 von Anfang an kritisch begleitet und kann inzwischen auf erste Lehr- und Handbücher verweisen, die einen vergleichsweise hohen Grad an dogmatischer Durchdringung dieses sich rasant entwickelnden Rechtsgebiets erkennen lassen. ${ }^{6}$

Noch im 19. Jahrhundert und im ersten Teil des 20. Jahrhunderts be- deutete dem Völkerrecht die staatliche Souveränität alles, das Individuum hingegen wenig. Einzelpersonen waren als Völkerrechtssubjekte nicht anerkannt, d.h. sie waren völkerrechtlich weder berechtigt noch verpflichtet. Der Krieg wurde weithin als legitime Sanktion im zwischenstaatlichen Rechtsverkehr angesehen, und die dualistischen Theorien zum Verhältnis von Völker- und Landesrecht hatten den Primat des (national)staatlichen Rechts besiegelt. Dementsprechend konnte Heinrich Triepel 1899 feststellen: »Denn was der Staat befiehlt, gilt es zu wissen. Gegen sein Gebot, nicht gegen das des Völkerrechts sündigt der Untertan. $«^{7}$ Kurzum: Es gab weder eine Anerkennung von Menschenrechten noch ihren völkerstrafrechtlichen Schutz. Heute stellt sich die kriminalpolitische Ausgangssituation grundlegend anders dar: Abgesehen von der fortschreitenden Internationalisierung des Rechts sind es maßgeblich die Menschen-rechte und Unternehmungen zu ihrem Schutz auf nationaler und internationaler Ebene, die die nationale Souveränität zunehmend durchlöchern. Mit dem Inkrafttreten des Römischen Statuts von $1998^{8}$ zum 1.7.2002 existiert in Den Haag erstmals ein ständiger Internationaler Strafgerichtshof. Das Statut ist inzwischen von 139 Staaten unterzeichnet und von 99 Staaten ratifiziert worden. ${ }^{9}$ Der deutsche Gesetzgeber hat es durch die Schaffung eines eigenen Völkerstrafgesetzbuches (VStGB) ${ }^{10}$ umgesetzt. Die Arbeitsfähigkeit des Internationalen Strafgerichtshofs ist seit dem Frühjahr 2003 hergestellt. Erste Ermittlungen betreffen Ereignisse in der Demokratischen Republik Kongo, in Uganda und im Sudan ${ }^{11}$. Zwar ist das weitere Schicksal dieses »Weltstrafgerichts " ungewiss - nicht zuletzt wegen des Wider-standes, den ihm die Vereinigten Staaten von Amerika unter Präsident Bush entgegen setzen. Doch die breite Zustimmung aus aller Welt, die sich in ei- nem überraschend schnellen Ratifizierungsprozess manifestiert, stimmt hoffnungsvoll. Jedenfalls gibt es nun erstmals eine permanenete internationale Strafgerichtsbarkeit, die die nationale im Wege der Komplementarität (Art. 17 Rom-Statut) ergänzt. Es ist dies nicht der Ort, um die lange Entwicklung, die u.a. über die Nürnberger Nachkriegsprozesse sowie über die ad-hoc-Tribunale der Vereinten Nationen für das ehemalige Jugoslawien und für Ruanda geführt hat, rechtshistorisch nachzuzeichnen oder gar nach den ideengeschichtlichen Voraussetzungen $\mathrm{zu}$ fragen. ${ }^{12}$ Im Folgenden soll vielmehr aus einem spezifisch kriminologischen Blickwinkel nach dem Beitrag gefragt werden, den die Kriminologie beizusteuern hätte.

\section{Makrokriminalität - ein Thema für die Kriminologie!}

Mit dem Begriff »Makrokriminalität « hatte Jäger in den 1980er Jahren vor allem Genozid, Kriegsverbrechen, Staatsterrorismus und nukleare Massenvernichtung ins Auge gefasst. Er beklagte an den Kriminalwissenschaften eine "Nahraum-Moral «, die die politische Sphäre nicht mehr erreiche und "gerade die schwersten und folgenreichsten Formen menschlichen Unrechts aus den kriminalwissenschaftlichen Denkkonzepten weitgehend ausgeblendet « habe. ${ }^{13}$ Ich verstehe "Makrokriminalität « gleichfalls als Konzept, das keine Schnittmenge mit Wirtschaftsdelikten bildet und eigenständig neben die von wirtschaftlich Mächtigen verübte Kriminalität tritt. Unter den weiter gefassten Oberbegriffen "Kriminalität der Mächtigen « bzw. »Staatskriminalität« bezeichnet »Makrokriminalität« eine besonders beunruhigende Form einer kriminellen Politik, die von Staatsorganen und/ oder staatlichen Stellen (z.B. Staatspräsident, Regierungschef, Militär, Polizei oder Geheimdienste) aus- geht, sich gezielt gegen Leben, Leib und Freiheit von Individuen richtet und als Menschenrechtsverbrechen adäquat erfasst werden kann. ${ }^{14}$ Das Präfix »Makro« kennzeichnet dabei nicht nur, gleichsam auf der Tatbestandsseite, die Dimension der Tat, das Großverbrechen, welches schwerste Schäden anrichtet und in das staatliche Stellen involviert sind. Auf der Rechtsfolgenseite steht es für die außerordentlichen Anstrengungen, die aufgebracht werden müssen, um eine Strafverfolgung von Makrokriminellen zu erreichen, die sich aufgrund ihrer Machtpositionen gegen nationale Strafverfolgung absichern und sich ihr entziehen. Die Erfahrungen aus der Vergangenheit lassen sich insoweit thesenartig zusammenfassen ${ }^{15}$ :

- Verbrechen, die durch den Staat selbst begangen oder organisiert werden, fordern ungleich mehr Opfer als »normale« Kriminalität.

- Staatliche Kriminalität ist gefährlicher als "normale« Kriminalität, weil der Staat über das Medium des Rechts und da, wo Rundfunk, Fernsehen und Druckmedien gleichgeschaltet sind, auch über die Sprache verfügt. Die psychischen Folgen für die Opfer sind so gravierend, weil das Unrecht systematisch geleugnet wird.

- Totalitäre Staaten ohne ein System von Gewaltenkontrolle sind besonders anfällig für staatliche Verbrechen.

- Voraussetzung eines effektiven Schutzes vor staatlichen Verbrechen ist die Anerkennung von Menschenrechten.

- Strafrecht und Kriminologie müssen bei Staatsverbrechen ihr Kriminalitätsverständnis erweitern und auf einen Verbrechensbegriff zurückgreifen, der sich seine Unabhängigkeit von den Vorgaben des nationalen Rechts bewahrt.

- Auf die nationale Justiz ist bei der Verfolgung völkerrechtlicher Verbrechen kein Verlass, jedenfalls dann nicht, wenn eigene Staatsangehörige betroffen sind. Das haben u.a. die Kriegsverbrecherprozesse vor dem deutschen Reichsgericht in Leipzig 1920/21, die amerikanische Haltung zu Vietnam und zahlreiche Staaten in Südamerika (z.B. Chile und Guatemala) belegt.

- Nicht der Mangel an völkerstrafrechtlichen Vorschriften hat der Anwendung des Völkerstrafrechts 
entgegengestanden, sondern der Ost-West-Gegensatz (bis 1989) und mangelnder politischer Wille.

- Strafrechtliche Verfahren dienen der Kanalisierung sozialer Konflikte; sie verfolgen das Ziel der Befriedung durch Recht. Das gilt auch für Strafverfahren wegen völkerrechtlicher Verbrechen. Die Alternativen $\mathrm{zu}$ einem zivilisierten rechtlichen Verfahren wären Krieg, Vergeltung bzw. Rache oder kollektive Haftung.

\section{Viktimologie und Straflosigkeit}

Viktimologischen Forschungen verdanken wir weitreichende Einsichten in die Belastungen, denen Opfer von Straftaten ausgesetzt sind. Besonders nach Gewaltdelikten leiden sie, abgesehen von den physischen Tatfolgen, regelmäßig unter Depression, Angst, der Infragestellung der Zukunftsperspektive, unter somatischen Symptomen, Verbrechensfurcht und belastendem Vermeideverhalten. Ausgelöst durch die Erfahrung von Kontrollverlust und eigener Verletzlichkeit ändern sie nach der Tat ihr Welt- und Selbstbild, sie sind in ihrem Vertrauen und Selbstwertgefühl erschüttert und haben ein starkes Bedürfnis nach formeller und informeller sozialer Unterstützung. ${ }^{16}$ In zahlreichen Fällen wird eine posttraumatische Belastungsstörung diagnostiziert. Diese Krankheit ist seit 1983 offiziell anerkannt und wird sowohl im Diagnostic and Statistical Manual of Mental Disorders (DSM IV) als auch in der International Classification of Diseases (ICD-10) als eigene Kategorie einer psychischen Störung aufgeführt. Als die drei zentralen Symptomgruppen werden intrusives Wiedererleben (z.B. in Tag- oder Albträumen), ausgeprägtes Vermeideverhalten und Hypersensibilität (z.B. Unfähigkeit zur Entspannung, Schreckhaftigkeit, Übererregbarkeit) genannt. Die Betroffenen sind zum Teil psychisch erstarrt, sie fühlen sich und der Welt um sie herum entfremdet und verlieren nicht selten das Interesse an zuvor gerne ausgeübten Tätigkeiten. ${ }^{17}$ Auch mittelbare Opfer, z.B. Intimpartner, Familienangehörige, können ähnliche Persönlichkeitsschäden davontragen. Bei ihnen treten vor allem Gefühle der Machtlosigkeit, der Verwundbarkeit und der
Schuld auf, weil sie es als eigenes Versagen empfinden, dass sie keinen Schutz gewähren konnten. ${ }^{18}$

Opfer mit schweren psychischen Tatfolgen wünschen sich in erster Linie Krisenintervention, persönliche Hilfe, Solidarität und normative Klarstellung. Ihre Reaktionen sind insoweit wenig punitiv. Auch wenn später, bei Inhaftierung des Täters, eine subjektive Genugtuungsfunktion von Strafe feststellbar ist, geht es diesen Opfern in der Regel weder um Rache noch um Vergeltung. ${ }^{19}$ Werden Straftatopfer in diesen Erwartungen durch ihren sozialen Nahraum oder durch die Instanzen der formellen Sozialkontrolle enttäuscht, weil beispielsweise Polizeioder Justizangehörige ihnen keinen Glauben schenken oder ihnen eine Mitschuld am Geschehen zuschreiben, so wird der Opferstatus verfestigt. Es handelt sich um eine sekundäre Viktimisierung, deren Konsequenzen schwerwiegender sein können als die unmittelbaren Tatfolgen. ${ }^{20}$ In jedem Fall steigt dadurch das Risiko, dauerhaft unter einer posttraumatischen Belastungsstörung zu leiden. Umgekehrt ist diese Störung weniger gravierend, je positiver das Opfer die interaktionale Gerechtigkeit im Strafverfahren einschätzt. ${ }^{21}$ Das Opfer ist aber nicht nur besonders verletzlich; im Hinblick auf weitere Viktimisierung ist es auch besonders gefährdet. Von Seiten des Täters droht dem Opfer eine Neutralisierung der Tat, die das Opfer herabsetzt bzw. verantwortlich macht (blaming the victim). Und selbst unbeteiligte Beobachter können der Versuchung erliegen, um die Erschütterung des Weltverständnisses (belief in a just world) durch das Verbrechen rationalisierend $\mathrm{zu}$ verleugnen, den vorwurfsvollen Blick vom Täter auf das Opfer zu lenken (»mir wäre das nicht passiert«).

Bei Makrokriminalität sehen sich die Opfer einem übermächtigen (Unrechts-)Staat gegenüber, der seine Verbrechen organisiert, systematisch, und wiederholt verübt. Wegen der Intensität des Unrechts und der Wehrlosigkeit der Opfer sind die Schädigungen besonders gravierend. Die Asymmetrie der politischen Machtverhältnisse und der privilegierte Zugang des Staates zu den Medien der Sprache (z. B. Propagan- daapparat) und des Rechts führen dazu, dass das begangene Unrecht systematisch geleugnet wird und keine Aussicht auf gerichtliche Aufklärung oder gar Bestrafung der Verantwortlichen besteht. Auf diese Weise bleibt das Individuum mit seiner Wahrheit alleine und kann sich in der Öffentlichkeit nicht gegen die offizielle Version des Geschehens behaupten. Ein internationales Gericht ist insofern der geeignete Ort für ein Strafverfahren gegen Makrokriminelle, weil diese auf nationaler Ebene keine Strafverfolgung zu befürchten haben. Die Rechtsverweigerung kann dort vielfältige Formen annehmen. Ob faktisch nicht ermittelt, angeklagt oder verurteilt wird oder ob die Straflosigkeit per Gesetz abgesichert wird - im Wege der Ausnahmegesetzgebung, der Immunität oder der Amnestie: Politisches Leitmotiv ist die Impunität, also die Straflosigkeit der Täter. ${ }^{22}$ Diese ist fatal, weil sie potentiellen Tätern Freiräume und Tatanreize schafft. Bekanntlich wird Makrokriminalität nicht nur, ja nicht einmal zum größeren Teil durch Menschen begangen, die zu den Verbrechen gezwungen werden. Vielmehr sind häufig persönliche und wenig schmeichelhafte Motive ausschlaggebend, zum Beispiel Fanatismus, Opportunismus, vorauseilender Gehorsam, berufliche Aufstiegswünsche, kriminelle Nebenmotive (z. B. bei Plünderungen) oder bloße Gleichgültigkeit. ${ }^{23}$ Anders wären auch sog. Exzesstaten, die noch über das angeordnete Verhalten hinausgehen, schwerlich zu erklären. Kurzum: Straflosigkeit schafft Handlungsspielräume, sie erzeugt ein Kli$\mathrm{ma}$, in dem die Normverletzung zur Normalität wird und »rechtens « erscheint, und sie begünstigt damit das Erlernen und Verfestigen solcher Verhaltensweisen.

\section{Menschenrechte und Schwereeinschätzung}

In jüngerer Zeit wird mit zunehmender Deutlichkeit herausgestellt, dass die Straflosigkeit schwerer Menschenrechtsverletzungen eine erneute Verletzung der Menschenrechte der geschädigten Person darstellt und mit Schutz- und Strafpflichten des Staates nicht in Einklang zu bringen ist. Der Menschenrechtsausschuss der Vereinten Nationen, der über die Einhaltung des Internatio- nalen Pakts über bürgerliche und politische Rechte vom 19.12.1966 wacht, hat dazu erklärt, dass »amnesties are generally incompatible with the duty of States to investigate such acts; to guarantee freedom from such acts within their jurisdiction; and to ensure that they do not occur in the future. States may not deprive individuals of the right to an effective remedy including compensation and such full rehabilitation as may be possible. $\ll^{24}$ Allgemeine Opferrechte wie das Recht auf einen wirksamen Rechtsbehelf (right to an effective remedy) und das einklagbare Recht auf Wiedergutmachung (enforcable right to compensation) werden spezifiziert durch die Resolution 40/34 der Generalversammlung der Vereinten Nationen aus dem Jahre 1985. Diese Declaration of Basic Principles of Justice for Victims of Crime and Abuse of Power ist völkerrechtlich nicht verbindlich; aus Sicht der Vereinten Nationen formuliert sie aber Grundprinzipien für den Umgang mit Verbrechensopfern. Unter anderem wird ein Anspruch auf materiellen, medizinischen, psychologischen und sozialen Beistand statuiert und ein eigener Abschnitt den Opfern von Machtmissbrauch gewidmet. ${ }^{25}$ Das Völkerrecht räumt inzwischen der Völkergemeinschaft und den einzelnen Staaten nicht nur die Befugnis ein, Völkerrechtsverbrechen im Wege des Universalitätsprinzips zu verfolgen; zumindest Tatortstaaten sind nach Völkervertragsrecht, zum Teil auch nach Völkergewohnheitsrecht, zur Bestrafung dieser Delikte verpflichtet. ${ }^{26}$ Spezielle Strafverfolgungspflichten ergeben sich etwa aus der Konvention über die Verhütung und Bestrafung des Völkermordes vom 9.12.1948, aus einzelnen Vorschriften der Genfer Konventionen von 1949 sowie aus dem Römischen Statut von 1998.

Immer noch fürchten und bekämpfen einige Staaten jede Einschränkung ihrer nationalen Souveränität infolge der Durchsetzung internationalen Rechts. Die Abneigung, die dem Internationalen Strafgerichtshof in diesen Teilen der Welt entgegenschlägt, ist ganz überwiegend machtpolitisch motiviert. Die Ablehnung einer internationalen Strafgerichtsbarkeit kann dabei, wie im Falle der USA unter Präsident Bush, ganz unverhohlen auftreten. 
Sie kann sich auch als Kritik westlichen Denkens und als Infragestellung der Universalität der Menschenrechte ausgeben. Sie kann aber keinesfalls darüber hinwegtäuschen, dass es sich bei der Errichtung des ständigen Internationalen Strafgerichtshofes um ein Projekt handelt, welches sich von Anfang an auf einen breiten, alle Kontinente und Kulturen umfassenden Konsens stützen konnte. Der Anstoß ging 1989 von einem Vorschlag Trinidad und Tobagos aus. In Rom erhielt das Statut bei der Schlussabstimmung - bei 21 Enthaltungen - 120 Ja-Stimmen und nur 7 Gegenstimmen (vermutlich USA, Israel, China, Irak, Jemen, Katar und Libyen). Inzwischen hat rund die Hälfte aller Staaten der Welt das Statut ratifiziert, noch mehr haben es gezeichnet. Die 18 Richter, die im Februar 2003 von der Versammlung der Vertragsstaaten gewählt wurden, kommen neben England, Frankreich, Kanada und Deutschland u.a. aus Bolivien, Mali, Trinidad und Tobago, Costa Rica, Ghana, Südafrika, Lettland, Korea und Samoa. Und auch das Engagement beispielsweise der afrikanischen Staaten wie auch von Nichtregierungsorganisationen aus aller Welt ist ein eindrucksvoller Beleg dafür, dass der internationale Menschenrechtsdiskurs viel weiter fortgeschritten ist, als der platte Einwand eines westlichen Eurozentrismus glauben machen will. Die breite Unterstützung bestätigt aber auch die empirisch abgesicherte Erkenntnis der Kriminologie, dass es - gleichsam als Spiegelung der Debatte um die Universalität der Menschenrechte - ein hohes Maß an weltweiter Übereinstimmung in der Schwereeinschätzung von Delikten gibt. In zahlreichen Studien ließ sich feststellen, dass, und zwar interkulturell, Mord und Totschlag am schwersten eingeschätzt werden, es folgen die Sexualdelikte, die schweren Körperverletzungen und der Raub, sodann Betrug und Diebstahl. ${ }^{27}$

\section{Individuelle strafrechtliche Verantwortlichkeit}

Die Justiz, gleich ob nationale oder internationale, kann die begangenen Verbrechen weder ungeschehen machen noch kann sie die seelischen Verwundungen heilen, die die Opfer erlitten haben. Das ist evident.
Aber die Justiz kann dazu beitragen, dass dem Opfer kein weiterer Schaden zugefügt wird, dass es als Subjekt behandelt wird und seine Wahrheit erzählen kann, dass Neutralisierungen entgegengewirkt wird und Tatsachen dokumentiert werden. Insofern gehen die justiziellen Leistungen über jene bei allgemeiner Kriminalität hinaus. Besonders im internationalen Strafrecht wächst der Justiz eine Bedeutung zu, die ich eine soziale Funktion nennen möchte und die die Aspekte Konfliktregelung, Prävention, Dokumentation und Opferschutz umfasst.

Die Kanalisierung eines Konflikts durch eine internationale Strafgerichtsbarkeit hat gegenüber früheren Formen der Sanktionierung im Völkerrecht den Vorzug der friedlichen Konfliktregelung, der Verbrechensprävention sowie der Gleichförmigkeit und Unabhängigkeit der Rechtsanwendung. Die individuell Schuldigen verantwortlich zu machen, ist ein großer Fortschritt und begünstigt die Ersetzung alter Machteliten im Prozess des gesellschaftlichen Neuanfangs. Der mit dem Strafen verfolgte Zweck besteht hauptsächlich in der Generalprävention. Dieser Strafzweck will in seiner negativen Variante potentielle Täter von der Verbrechensbegehung abhalten und in seiner positiven Variante alle Normadressaten in ihrem Vertrauen in die Unverbrüchlichkeit der Rechtsordnung bestärken. ${ }^{28}$ Hier ruhen in erster Linie die Hoffnungen, die in eine funktionierende internationale Strafgerichtsbarkeit gesetzt werden. Denn wenn eine Abschreckungswirkung nicht von hohen Strafdrohungen, sondern maßgeblich von der Existenz eines Strafrechts und eines effektiven Strafverfolgungssystems ausgeht, bestehen mit der erstmaligen Einrichtung eines ständigen Internationalen Strafgerichtshofes durchaus Chancen für eine negative Generalprävention. Gleichwohl dürfte der Akzent auch in Zukunft mehr auf der Integrationsprävention liegen. Denn gerade im internationalen Strafrecht erscheint es besonders dringlich, ein entsprechendes Normbewusstsein und Normvertrauen erst $\mathrm{zu}$ schaffen. Der Strafhöhe kommt in diesem $\mathrm{Zu}$ sammenhang eine untergeordnete Rolle $\mathrm{zu}$, sofern nur ein allgemeines Strafverfolgungs- und Bestrafungsrisiko gewährleistet bleibt.
Darüber hinaus verfolgt das internationale Strafrecht auch das Ziel der Solidarität mit dem Opfer und der Dokumentation historischer Fakten. Das sind justizielle Leistungen, die über die bloße Verbrechensprävention hinausgehen. Unter Solidarität mit dem Opfer verstehe ich Aspekte des Opferschutzes, die als Anerkennung des Opferstatus oder als Vermeidung von Viktimisierungsschäden bezeichnet werden können. Im Falle eines Schuldspruchs des Täters wird dem Opfer attestiert, dass ihm nicht ein zeitgeschichtliches Unglück widerfahren ist, sondern dass ihm Unrecht ange$\tan$ wurde. ${ }^{29}$ Ein solcher Schuldspruch behält seine Bedeutung auch, wenn ein Täter seine Strafe nicht verbüßen muss, z. B. weil er zu alt oder zu krank ist. Mit einem Urteil werden außerdem geschichtliche Sachverhalte klargestellt. Das dient, im Sinne des Opfers wie auch der Gesellschaft, der Vermeidung von Folgeauseinandersetzungen; denn das Urteil kann künftigen geschichtsrevisionistischen Bestrebungen und allen Versuchen, das begangene Unrecht zu neutralisieren, entgegengehalten werden.

Die Ermittlung der Tathintergründe und derjenigen, die die Verantwortung für das Verbrechen tragen, übernehmen für den Bewältigungsprozess des Tatopfers eine wichtige Rolle. Für Kriminologie und Strafrecht gleichermaßen stellt sich in diesem Zusammenhang allerdings die Frage, wie groß die Handlungsspielräume des Einzelnen bei der Begehung von Systemunrecht tatsächlich sind, inwieweit also der strafrechtliche Schuldvorwurf eine empirische Basis im Sinne eines Anders-Handeln-Könnens aufweist. Was in Kriminologie und Geschichtswissenschaft an empirischen Daten verfügbar ist, deutet eher auf ausreichende Handlungsspielräume für Individuen in Entscheidungssituationen hin. In der Nachkriegszeit ist in Deutschland die Vorstellung weit verbreitet gewesen, dass es nur unter Gefährdung des eigenen Lebens möglich gewesen sei, die Ausführung eines Tötungs- oder Vernichtungsbefehls zu verweigern. Dazu trug bei, dass der sog. Befehlsnotstand in undifferenzierten und polemischen Auseinandersetzungen vor Gerichten, in Politik und Publizistik zum apologe- tischen Topos verkam. ${ }^{30}$ Unter hunderten von Fällen, die die Zentrale Stelle der Landesjustizverwaltungen zur Aufklärung von NS-Verbrechen in Ludwigsburg untersuchte, ließ sich nicht ein einziger finden, in dem ein Militärangehöriger, der die Ausführung eines verbrecherischen Befehls ablehnte, selbst exekutiert oder ernsthaft bestraft worden wäre. ${ }^{31}$ Jägers kriminologische Analyse von 103 Fällen, in denen sich nach Zeugenaussagen Funktionäre Tötungsbefehlen widersetzt oder entzogen haben, hat in keinem Fall eine Schädigung an Leib oder Leben wegen Befehlsverweigerung, insbesondere durch Todesurteil, Erschießung oder Einweisung in ein Konzentrationslager, nachweisen können. Soweit die Befehlsverweigerer Nachteile hinnehmen mussten, handelte es sich überwiegend um Versetzungen an die Front, Rügen und - im Falle von Offizieren - auch um Degradierungen. Allerdings zogen Verweigerungen in beträchtlichem Maße überhaupt keine nachteiligen Folgen nach sich. ${ }^{32}$ Neuere geschichtswissenschaftliche Studien untermauern diese Sicht. ${ }^{33}$

Empirische Befunde schließen also die Annahme individueller strafrechtlicher Verantwortlichkeit nicht aus. Im Gegenteil: Aus den Faktoren, die Gehorsams- bzw. Ungehorsamsreaktionen begünstigen, können wichtige sachliche Gesichtspunkte für die strafrechtliche Behandlung der Täter gewonnen werden, nämlich von erhöhter individueller Verantwortlichkeit bis hin zu entschuldigungsähnlichen Konstellationen. Gerade aus deutscher Sicht ist vor der »Suggestion « zu warnen, »kollektive Verbrechen als das verselbständigte Handeln von ,Systemen', ,Apparaturen' und organisierten Großgruppen zu interpretieren, das für persönlich motivierte Verhaltensweisen keinen Raum läßt. «34 Zwar zeigt die sozialpsychologische Forschung, dass Menschen dazu neigen, ihre eigene Fähigkeit und die anderer zu autonomem Handeln zu überschätzen, während sie mehrheitlich in konkreten Drucksituationen auch ohne Androhung von Sanktionen zu konformem Verhalten gebracht werden können..$^{35}$ Dennoch muss eine Kriminologie der Makrokriminalität zur Kenntnis nehmen, dass individuelles Unrecht nicht etwa im systemischen Unrecht auf- 
geht, also gleichsam dahinter verschwindet, sondern dass unter den Bedingungen systemischen Unrechts individuelles Unrecht in zahlreichen Facetten ermöglicht und gefördert wird.

Vereinzelt werden auch Modelle einer kollektiven strafrechtlichen Verantwortlichkeit erörtert. Aber eine Strafe, welche darauf aus wäre, das ganze Unrecht des Systems zu sühnen, geriete zwangläufig in die Gefahr der Kollektivschuldzuweisung. Das schon in Nürnberg restriktiv gehandhabte Modell von Organisationsverbrechen hat zu Recht keinen Eingang in das Völkerrecht gefunden. Gegen eine kollektive strafrechtliche Haftung spricht insbesondere, dass das Prinzip der individuellen völkerstrafrechtlichen Verantwortlichkeit empfindlich geschwächt würde. Die Verschiebung der Verantwortung auf ein böses System würde sozialpsychologisch einen Rückschritt bedeuten und eine Haltung begünstigen, die makro-kriminelle Verhaltensweisen und ihre strafrechtliche Ahndung in den Bereich der Politik verweist und das Recht für nicht zuständig erachtet. Sie eröffnet ohne Not das Risiko der Instrumentalisierung des Rechts durch die Politik und kollidiert überdies mit dem Grundsatz der Unschuldsvermutung.

\section{Wahrheitskommissionen}

Als mögliche Alternative zu strafrechtlichen Verfahren ist zuweilen von sog. Wahrheitskommissionen die Rede, die die Palette der gesellschaftlichen Reaktionsmöglichkeiten auf Makrokriminalität und Menschenrechtsverbrechen erweitern. Die Erfahrungen mit ihnen sind jedoch ernüchternd. So kann schon das Mandat einer "Wahrheits«- oder Untersuchungskommission so beschnitten werden, dass bestimmte Geschehnisse nicht untersucht werden. Man kann Informationen zurückhalten, der Kommission Zwangsmittel verwehren, die für die Gewinnung von Informationen unentbehrlich sind, die Abfassung und Veröffentlichung des Abschlussberichts hintertreiben oder die Nennung von Namen untersagen. Als letztes Mittel lässt sich schließlich die Arbeit einer Kommission dadurch konterkarieren, dass man die von ihr festgestellten Verbrechen nachträglich amnestiert. ${ }^{36}$ Die in die Kommissionen gesetzten Erwartungen werden infolge einer meist einseitigen Versöhnungsbereitschaft der Opfer (bzw. ihrer Angehörigen) sowie ihrer unzureichenden Betreuung regelmäßig nicht erreicht. Ein bemerkenswerter Sonderfall ist die »Truth and Reconciliation Commission « in Südafrika, die nicht jenseits des Strafrechts operierte, sondern durch ihre Befugnis zur Amnestierung im Einzelfall mit den Strafverfahren verzahnt war. $\mathrm{Zu}$ Recht baute sie darauf, dass denen, die keine Einsicht zeigen, erst die Drohkulisse des Strafrechts Zugeständnisse abringen wird. In der Tat hat nur tatsächliche oder drohende Strafverfolgung die meisten Angehörigen der südafrikanischen Sicherheitskräfte veranlasst, einen Amnestieantrag $\mathrm{zu}$ stellen. "Die tatsächliche und ernsthafte strafrechtliche Verfolgung von Staatskriminalität war daher eine Funktionsbedingung des Amnestiemodells. «37 Gleichwohl wird die Bedeutung des südafrikanischen Modells quantitativ wie qualitativ häufig überschätzt. Die Zahl der Anträge auf Amnestierung, die mit der Bereitschaft zu umfassender Offenlegung der Tatumstände verbunden sein musste (full disclosure), blieb weit hinter den Erwartungen zurück und war vor allem bei den Angehörigen des alten Sicherheitsapparates gering. Vermutlich bauten viele darauf, es werde den Vertretern der alten Eliten gelingen, Ammestierungen durchzusetzen; die gesellschaftliche Debatte um Generalamnestien geht in Südafrika jedenfalls weiter. In ihrem Abschlussbericht bemängelte die Kommission u.a., dass ihr eine Kooperation bei der Aufklärung der Verbrechen durch die alten Eliten bei der Polizei, den Streitkräften, den Geheimdiensten und der Staatsanwaltschaft bis zuletzt verweigert wurde..$^{35}$

Die Vorstellung, »Wahrheitskommissionen « könnten, sei es auf nationaler, sei es auf internationaler Ebene, künftig die Aufgaben einer internationalen Strafgerichtsbarkeit übernehmen, ist für eine Kriminologie, die Opferinteressen und »harm reduction « bei der Sanktionierung in eine Balance zu bringen versucht, verlockend. Trotz positiver Ansätze repräsentiert die südafrikanische Wahrheits- und Versöhnungskom- mission jedoch kein verallgemeinerungsfähiges Modell. Eine Optimierung ist mit Blick auf künftige Kommis-sionen nicht ausgeschlossen. $\mathrm{Zu}$ den Minimalanforderungen könnte gehören, dass eine Amnestie bei schwersten Verbrechen wie Genozid oder Mord (jedenfalls für die Führungsebene) ausgeschlossen ist, dass bei Ablehnung der Amnestierung ein Strafverfahren nicht durch ein generelles Beweisverwertungsverbot verhindert wird, dass die psychotherapeutische Betreuung von Opfern und ihren Angehörigen sichergestellt ist und dass ihr Beitrag zum Versöhnungsprozess dadurch in den Vordergrund gerückt wird, dass sie analog zu den erfolgreich praktizierten Formen der Mediation im Strafrecht (Täter-Opfer-Ausgleich) - dem Amnestievorschlag der Kommission in der Regel zustimmen müssen. Die Legitimation von Amnestierungen würde so in sehr markanter Weise auf eine neue Grundlage gestellt. Nicht mehr der Staat bzw. die Kommission als sein Organ würden die Amnestie aus Gründen der - mehr oder weniger gut gemeinten - Staatsräson verordnen, sondern der in seinen Rechten selbst Betroffene bzw. seine Angehörigen würden die Basis schaffen für gesellschaftliche Aussöhnung.

\section{Schluss}

Die Kriminologie steht bei der Thematik der internationalen Strafgerichtsbarkeit vor einer Fülle offener Forschungsfragen. Ihr Beitrag zur naturgemäß internationalen - kriminal-wissenschaftlichen Diskussion kann sowohl theoretischer als auch empirischer Natur sein. Die Ausweitung des Kriminalitätsverständnisses bringt es mit sich, dass praktisch alle bisher relevanten kriminologischen Fragen auf Erscheinungen der Makrokriminalität bezogen werden können. So sind Forschungen zu Fragen der Phänomenologie und Ätiologie der Makrokriminalität ebenso nötig wie durch den labeling-Ansatz inspirierte Fragestellungen, beispielsweise zur Definition von makrokriminellen Verhaltensweisen durch internationale Ermittlungsstellen bzw. Strafgerichtshöfe und durch die Politik. Die Stichworte Selektivität der Rechtsanwendung, Einstellungen der Verfahrensbeteiligten, Kommunikation im Strafprozess, Wir- kung von Sanktionen und menschenrechtliche Problemfelder mögen hier beispielhaft die Ausmaße dieser terra incognita andeuten, vor der sich die Kriminologie gestellt sieht.

Priv.-Doz. Dr. Frank Neubacher M.A., Institut für Kriminologie der Universität zu Köln hat zu diesem Thema habilitiert.

\section{Fußnoten:}

$1 \mathrm{Vgl}$. etwa Schwind, Kriminologie, 15. Aufl. 2005, S. 8.

2 Engl:: Crimes against Humanity; die deutsche Übersetzung ist unglücklich, hat sich aber durchgesetzt. Vgl. Mans$k e$, Verbrechen gegen die Menschlichkeit als Verbrechen an der Menschheit, 2003.

3 Das Verbrechen der Aggression, über welches der Gerichtshof seine Jurisdiktion vorerst nicht ausüben kann (s. Art. 5 Abs. 2, Art. 123 Rom-Statut), klammere ich aus, dazu Hummrich, Der völkerrechtliche Straftatbestand der Aggression, 2001.

4 Vgl. Jäger, Makrokriminalität, Studien zur Kriminologie kollektiver Gewalt, 1989.

5 Vgl. Möller, Völkerstrafrecht und Internationaler Strafgerichtshof - kriminologische, straftheoretische und rechtspolitische Aspekte, 2003; Reese, Großverbrechen und kriminologische Konzepte - Versuch einer theoretischen Integration, 2004; Neubacher, Kriminologische Grundlagen einer internationalen Strafgerichtsbarkeit. Politische Ideen- und Dogmengeschichte, kriminalwissenschaftliche Legitimation, strafrechtliche Perspektiven, 2005.

6 Vgl. Ambos, Der Allgemeine Teil des Völkerstrafrechts, 2002; Werle, Völkerstrafrecht, 2003; Satzger, Europäisches und Internationales Strafrecht, 2005.

7 Triepel, Völkerrecht und Landesrecht, 1899, S. 333.

8 Rome Statute of the International Criminal Court, s. BGBl. 2000 II, S. 1393. 9 Stand: 7.7.2005.

10 Vom 26.6.2002 (BGBl. 2002 I, S. 2254), in Kraft seit dem 30.6.2002. Dazu Satzger, Das neue Völkerstrafgesetzbuch - Eine kritische Würdigung, in: NStZ 2002, S. 125 ff.; Keller, Kritik des Völkerstrafgesetzbuches, in: NK 2003, S. $112 \mathrm{ff}$.

11 Infolge der Überweisung durch den UN-Sicherheitsrat, s. Resolution 1593 vom 29.6.2005. 
12 Dazu Neubacher, Kriminologische Grundlagen einer internationalen Strafgerichtsbarkeit, 2005; Ahlbrecht, Geschichte der völkerrechtlichen Strafgerichtsbarkeit im 20. Jahrhundert, 1999.

13 Jäger, Makrokriminalität, S. 13; ähnl. Jäger, Versuch über Makrokriminalität, in: Strafverteidiger 1988, S. 172, 175.

14 Vgl. Neubacher, Politik und Verbrechen - Zur Terminologie und Typologie staatlicher bzw. gegen den Staat gerichteter Kriminalität, in: MschrKrim 2002, S. 290 ff.

15 Weiter ausgeführt in: Neubacher, Tribunal der Vernunft oder hilfloser Versuch einer Verrechtlichung? - Acht Thesen zum ständigen Internationalen Strafgerichtshof, in: Recht und Politik 2003, S. $160 \mathrm{ff}$.

16 Siehe H.J. Schneider, Der gegenwärtige Stand der Opferforschung, in: MschrKrim 1998, S. 327; Orth, Strafgerechtigkeit und Bewältigung krimineller Viktimisierung, 2001, S. 19 f.

17 Vgl. Davison/Neale, Klinische Psychologie, 3. Aufl. 1988, S. 192; Yule (ed.), Post-Traumatic Stress Disorders: Concepts and Therapy, 1999.

18 H.J. Schneider, MschrKrim 1998, S. 329.

$19 \mathrm{Vgl}$. Kilchling, Opferinteressen und Strafverfolgung, 1995, S. 630, 673; Baurmann/Schädler, Das Opfer nach der Straftat - seine Erwartungen und Perspektiven, 1991, S. 279; H.J. Schneider, MschrKrim 1998, S. 333.

20 Kiefl/Lamnek, Soziologie des Opfers, 1986, S. 239 ff.; H.J. Schneider, MschrKrim 1998, S. 329.

21 Orth, Strafgerechtigkeit und Bewältigung krimineller Viktimisierung, 2001, S. 126.

22 Vgl. Ambos, Straflosigkeit von Menschenrechtsverletzungen, Zur »impunidad « in südamerikanischen Ländern aus völkerstrafrechtlicher Sicht, 1997.

23 Siehe auch Jäger, Verbrechen unter totalitärer Herrschaft, in: Handwörterbuch der Kriminologie, 2. Aufl. 1975, Dritter Band, S. $458 \mathrm{ff}$.

24 Zit. nach Cassesse, On the Current Trends towards Criminal Prosecution and Punishment of Breaches of International Humanitarian Law, in: European Journal of International Law 1998, S. 6.

25 Dazu Möller, Völkerstrafrecht und Internationaler Strafgerichtshof, 2003, S. $548 \mathrm{ff}$.

26 Werle, Völkerstrafrecht, 2003, S. 71 ff., 76; Nerlich, Apartheidkriminalität vor Gericht, 2002, S. 1 f. Ob eine solche Verpflichtung auch Drittstaaten trifft, ist im Einzelnen noch ungeklärt.
$27 \mathrm{Vgl}$. Sellin/Wolfgang, The Measurement of Delinquency, 1964, S. 278 Evans/Scott, The Seriousness of Crime Cross-Culturally, in: Criminology 1984, S. 41, 50; Kania/Brand/Zimmermann/Walter, Die Einschätzung von Gewaltdelikten im europäischen Vergleich, in: MschrKrim 2003, S. 247 ff

$28 \mathrm{Vgl}$. Nemitz, Strafzumessung im Völkerstrafrecht, 2002, S. 165.

29 So auch Reemtsma, Das Recht des Opfers auf die Bestrafung des Täters - als Problem, 1999, S. 25 f.

$30 \mathrm{Vgl}$. Frei, Vergangenheitspolitik, Die Anfänge der Bundesrepublik und die NS-Vergangenheit, 1999, S. 129, 229.
31 So Schreiber, Deutsche Kriegsverbrechen in Italien. Täter, Opfer, Strafverfolgung, 1996, S. 48 m.w.N

32 Jäger, Verbrechen unter totalitärer Herrschaft, 1967, S. 94 ff., 120 ff.

33 Browning, Ganz normale Männer, Das Reserve-Polizeibataillon 101 und die »Endlösung « in Polen, 1999, S. 87 f., 98 ff., s. für das Völkerstrafrecht auch Ambos, Der Allgemeine Teil des Völkerstrafrechts, 2002, S. 863.

34 Jäger, Versuch über Makrokriminalität, in: Strafverteidiger 1988, S. 177.

35 Vgl. Neubacher/Walter, Sozialpsychologische Experimente in der Kriminologie, 2002.

36 Mit Beispielen (u.a. aus Chile, Guatemala, El Salvador) und weiteren Nachweisen s. Neubacher, Kriminologische Grundlagen einer internationalen Strafgerichtsbarkeit, 2005, S. $456 \mathrm{ff}$

37 Nerlich, Apartheidkriminalität vo Gericht, Der Beitrag der südafrikanischen Strafjustiz zur Aufarbeitung von Apartheidunrecht, 2002, S. 335

$38 \mathrm{Vgl}$. Kutz, Amnestie für politische Straftäter in Südafrika, Von der Sharpeville-Amnestie bis zu den Verfahren der Wahrheits- und Versöhnungskommission, 2001, S. 100.

\section{Perspektiven für den deutschen Strafvollzug}

\section{Bernd Maelicke}

\section{Veränderte gesellschafts- und sozialpolitische Rahmenbedingungen}

ie grundlegenden und rapiden Veränderungen in Staat und Gesellschaft wirken sich selbstverständlich auch auf den deutschen Strafvollzug aus:

Die Globalisierung führt zu Umbrüchen in einem bisher unbekannten und unerwarteten Ausmass. Innovation und Modernisierung erfassen alle gesellschaftlichen Bereiche. Der Wegfall der ideologischen und nationalen Grenzen führt zur totalen Verunsicherung. Prinzipiell alle bisher tragenden und unbestrittenen Werte und Normen sind in Frage gestellt. »Global Denken« ermöglicht sowohl Fortschritt wie Rückschritt - Orientierung an humanen Grundwerten und sozialen Mindeststandards, aber auch Übernahme inhumaner Zielvorstellungen und Neudefinition von Armut und verstärkter Ausgrenzung. »Lokal Handeln« ermöglicht Eingehen auf regionale und situationsspezifische Besonderheiten, bedeutet aber auch den zunehmenden Verzicht auf überregionale Wertorientierungen und verbindliche Mindeststandards.

Der weltweite Wettbewerb der Betriebe und Unternehmen ergreift auch die Staaten und Gesellschaften.
Gewinnorientierung und Wirtschaftlichkeit bestimmen mehr und mehr das Handeln und das Bewusstsein der Mitarbeiter und des Managements - in der Wirtschaft, bei staatlichen Organisationen, ja sogar bei Non-Profit-Organisationen. Effektivität und Effizienz, verbunden mit wirksamen betriebswirtschaftlichen Methoden, werden zu den dominanten Erfolgsfaktoren.

Der deutsche Sozialstaat befindet sich in einer strukturellen und nahezu unlösbaren Finanzkrise. Die deutsche Einheit, fehlendes Wachstum und die strukturelle Arbeitslosigkeit haben alle Sozialleistungssysteme an die Grenze der Insolvenz geführt. Die demographische Entwicklung führt zu weiteren Belastungen, für die weder Politik noch Wissenschaft überzeugende Lösungen anbieten können.

\section{Veränderte kriminalpoliti- sche Rahmenbedingungen}

Der Umbruch in Staat und Gesellschaft erfasst selbstverständlich auch die Kriminalpolitik. Verunsicherte Bürger und aggressive Medien setzen die Politik so unter Druck, dass ein regelrechter Wettbewerb darüber stattfindet, wer mehr Sicherheit versprechen kann - unabhängig davon, ob diese Versprechen einge- löst werden können und möglicherweise zu noch mehr Verunsicherung führen.

Das in den 70er und 80er Jahren vorhandene Reform-Bündnis zwischen der Praxis, der Wissenschaft, dem Gesetzgeber, der Rechtsprechung, den Medien und rationalen Kriminalpolitikern ist zerbrochen. Aggressive Medien und populistische und opportunistische Politiker bestimmen die öffentliche Diskussion und das Handeln. Fach- und Führungskräfte fühlen sich verunsichert und verlassen, fachliche Mindeststandards der Resozialisierung geraten ins Wanken.

Wegfall der Grenzen und verschärfte Gesetze führen zur Geissel der Überlegung in den Anstalten menschenunwürdige Unterbringung überlagert alle gutgemeinten Behandlungsabsichten. Weitere Frustration von Mitarbeitern und Gefangenen ist die Folge. Die Öffentlichkeit gewöhnt sich an Zustände, die grundgesetzwidrig sind.

\section{Wirkungsorientierte Steuerung}

Unabhängig von der geschilderten Umbruchsituation und bereits seit Jahrzehnten wissenschaftlich und in vielen Kommissionen vorbereitet gewinnt in der Fachdiskussion und in der Praxis das fachliche Ziel einer verstärkten Wirkungsorientierung in der ambulanten und stationären Resozialisierung immer mehr an Bedeutung. Welche Behandlungsziele können durch welche Methoden mit welchem Wirkungsgrad reali- 